\title{
The evolutionarily conserved function of TBR1 in controlling the size of anterior commissure in human and mouse brains
}

\author{
Yi-Ping Hsueh $\mathbb{D}^{1} \cdot$ Tsan-Ting Hsu ${ }^{1} \cdot$ Tzyy-Nan Huang ${ }^{1}$
}

Received: 12 February 2020 / Accepted: 24 March 2020 / Published online: 9 April 2020

(c) The Author(s), under exclusive licence to European Society of Human Genetics 2020

\section{To the Editor:}

We read with great interest the article by Nambot et al. [1], in which 25 unreported individuals with mutations in the $T B R 1$ gene were analyzed in great detail at molecular and anatomical levels and that also provides detailed clinical features of the patients. Since the patients carry de novo mutations, only one of their TBRI alleles is mutated. Among the 25 patients, seven individuals were carefully analyzed by magnetic resonance imaging (MRI). Interestingly, a specific white matter structure of the brain, the anterior commissure (AC), is either thin or absent from all seven of those individuals. Other morphological abnormalities—including dysplastic hippocampi and/or cerebral cortex, severe gyral anomalies, and microcephalyare present in only some of the patients. Thus, reduction of the $\mathrm{AC}$ is the most common anatomical feature shared by patients carrying different mutations in the TBRI gene, although the Nambot et al. study did not quantify AC size.

These results are significant to study of TBR1. In two previous studies using $\mathrm{TbrI}^{+/-}$mice, which mimic the condition of monoallelic mutations that result in early translational termination, small or absent ACs were identified upon hematoxylin and eosin staining, luxol fast blue/ cresyl violet staining and MRI analysis [2, 3]. Note that the $\mathrm{AC}$ has both anterior and posterior parts. In the first of those two studies, histological methods revealed that the posterior part of the AC is the most sensitive structure to Tbrl haploinsufficiency [2]. Later, in the second study, more comprehensive analyses using MRI supported the findings of the first study and further demonstrated that the anterior part

Yi-Ping Hsueh

yph@gate.sinica.edu.tw

1 Institute of Molecular Biology, Academia Sinica, Taipei 11529, Taiwan of the AC is also affected by Tbrl haploinsufficiency [3]. Apart from these two papers, another study used adenoassociated virus (AAV) for expression of YFP-tagged membrane protein to trace axonal projection via the AC. The results suggest that the two amygdalae in the two brain hemispheres do not project to each other via the $\mathrm{AC}$ in $\mathrm{Tbrl}^{+/-}$mice, which is in contrast to wild-type littermates [4]. Thus, the small or absent AC is the most critical phenotype of $\mathrm{TbrI}^{+/-}$mice. The patient study contributed by Nambot and colleagues corroborates these mouse studies, further indicating that the AC defects caused by TBR1 deficiency are evolutionarily conserved between human and mice.

This evolutionary conservation suggests an interesting potential clinical therapy. In $\mathrm{Tbrl}^{+/-}$mice, autism-like behaviors-including reduced social interaction, impaired associated memory, and cognitive inflexibility—can be ameliorated by D-cycloserine treatment, which is an NMDAR co-agonist used to activate neurons [2]. Since TBR1 deficiency in both mice and human results in the same anatomical deficits, i.e. small or absent AC, it is likely that D-cycloserine could also ameliorate the behavioral defects of patients harboring mutations of their TBRl gene. As Nambot et al. [1] also point out in the last paragraph of their discussion, AC deficits could be a useful diagnostic feature of TBR1 deficiency. D-cycloserine might be considered for the patients with defects in the AC. However, based on results published previously [2-4], Nambot and colleagues were incorrect to claim that "Hypoplasia/absence of the AC and hippocampal dysplasia were not previously reported in mice or humans with TBR1 alterations,....". In fact, AC deficiency in mouse brains was first reported six years ago [2].

\section{Compliance with ethical standards}

Conflict of interest The authors declare that they have no conflict of interest. 
Publisher's note Springer Nature remains neutral with regard to jurisdictional claims in published maps and institutional affiliations.

\section{References}

1. Nambot S, Faivre L, Mirzaa G, Thevenon J, Bruel AL, MoscaBoidron AL, et al. De novo TBRl variants cause a neurocognitive phenotype with ID and autistic traits: report of 25 new individuals and review of the literature. Eur J Hum Genet. 2020. https://doi.org/ 10.1038/s41431-020-0571-6.
2. Huang TN, Chuang HC, Chou WH, Chen CY, Wang HF, Chou SJ, et al. $\mathrm{Tbrl}$ haploinsufficiency impairs amygdalar axonal projections and results in cognitive abnormality. Nat Neurosci. 2014; $17: 240-7$.

3. Huang TN, Yen TL, Qiu LR, Chuang HC, Lerch JP, Hsueh YP. Haploinsufficiency of autism causative gene Tbrl impairs olfactory discrimination and neuronal activation of the olfactory system in mice. Mol Autism. 2019;10:5.

4. Huang TN, Hsu TT, Lin MH, Chuang HC, Hu HT, Sun CP, et al. Interhemispheric connectivity potentiates the basolateral amygdalae and regulates social interaction and memory. Cell Rep. 2019; 29:34-48.e34. 